\title{
Hamiltonian Formalism for Solving the Vlasov-Poisson Equations and Its Applications to Periodic Focusing Systems and the Coherent Beam-Beam Interaction
}

\author{
Stephan I. Tzenov and Ronald C. Davidson \\ Plasma Physics Laboratory, Princeton University, Princeton, New Jersey 08543
}

\begin{abstract}
A Hamiltonian approach to the solution of the Vlasov-Poisson equations has been developed. Based on a nonlinear canonical transformation, the rapidly oscillating terms in the original Hamiltonian are transformed away, yielding a new Hamiltonian that contains slowly varying terms only. The formalism has been applied to the dynamics of an intense beam propagating through a periodic focusing lattice, and to the coherent beam-beam interaction. A stationary solution to the transformed Vlasov equation has been obtained.
\end{abstract}

Submitted to the Physical Review Special Topics - Accelerators and Beams 


\section{INTRODUCTION}

The evolution of charged particle beams in accelerators and storage rings can often be described by the Vlasov-Maxwell equations ${ }^{1,2}$. At high energies the discrete-particle collision term $^{3}$ comprises a small correction to the dynamics and can be neglected. Radiation effects at sufficiently high energies for leptons can be a significant feature of the dynamics, and should normally be included in the model under consideration.

The Vlasov-Maxwell equations constitute a considerable simplification in the description of charged particle beam propagation. Nonetheless there are only a few cases that are tractable analytically ${ }^{1,2}$. Therefore, it is of the utmost importance to develop a systematic perturbation approach, able to provide satisfactory results in a wide variety of cases of physical interest.

Particle beams are subject to external forces that are often rapidly oscillating, such as quadrupolar focusing forces, RF fields, etc. In addition, the collective self-field excitations can be rapidly oscillating as well. A typical example is a colliding-beam storage ring device, where the evolution of each beam is strongly affected by the electromagnetic force produced by the counter-propagating beam ${ }^{4-6}$. The beam-beam kick each beam experiences is localized only in a small region around the interaction point, and is periodic with a period of one turn.

In this and other important applications, one is primarily interested in the long-time behavior of the beam, thus discarding the fast processes on time scales of order the period of the rapid oscillations. To extract the relevant information, an efficient method of averaging is developed in Sec. 2. Unlike the standard canonical perturbation technique ${ }^{7,8}$, the approach used here is carried out in a "mixed" phase space (old coordinates and new canonical momenta) $)^{9}$, which is simpler and more efficient in a computational sense. It should be pointed out that the formalism developed here is strictly speaking non-canonical and in general does not provide complete elimination of fast oscillating terms in the transformed Vlasov equation in the mixed variables. Nevertheless, such an elimination can be performed 
in the new Hamiltonian in the mixed variables. Moreover, if the distribution function is assumed to be an arbitrary function of the new time-independent Hamiltonian, it is a stationary solution of the nonlinear Vlasov equation in the mixed variables. The canonical perturbation method developed in this paper is further applied to intense beam propagation in a periodic focusing structure (Sec. 3) and to the coherent beam-beam interaction (Secs. 4 and 5). A coupled set of nonlinear integral equations for the equilibrium beam densities is derived.

To summarize, the effectiveness of the Hamiltonian formalism developed in the present paper is demonstrated in two particular examples. In the first example discussed in Sec. 3, the short-scale dynamics is contained in the external focusing force acting on the beam, while an essential feature of the coherent beam-beam interaction treated in Secs. 4 and 5 is the relatively fast variation of the collective interaction between the colliding beams. The simplicity in applying the Hamiltonian averaging technique is embedded in the use of mixed canonical variables. Instead of expanding the generating function and the new Hamiltonian in terms of the new canonical coordinates and momenta ${ }^{7,8}$ one has to simply solve the Hamilton-Jacobi equations order by order. It should be emphasized that the mixed variable Hamiltonian formalism can be used to derive amplitude equations, describing processes of formation of patterns and coherent structures in a number of plasma and beam systems in which collective processes are important.

\section{THE HAMILTONIAN FORMALISM}

We consider a $N$-dimensional dynamical system, described by the canonical conjugate pair of vector variables $(\mathbf{q}, \mathbf{p})$ with components

$$
\begin{aligned}
& \mathbf{q}=\left(q_{1}, q_{2}, \ldots, q_{N}\right), \\
& \mathbf{p}=\left(p_{1}, p_{2}, \ldots, p_{N}\right) .
\end{aligned}
$$

The Vlasov equation for the distribution function $f(\mathbf{q}, \mathbf{p} ; t)$ can be expressed as 
$\frac{\partial f}{\partial t}+[f, H]_{\mathbf{q}, \mathbf{p}}=0$

where

$[F, G]_{\mathbf{q}, \mathbf{p}}=\frac{\partial F}{\partial q_{i}} \frac{\partial G}{\partial p_{i}}-\frac{\partial F}{\partial p_{i}} \frac{\partial G}{\partial q_{i}}$

is the Poisson bracket, $H(\mathbf{q}, \mathbf{p} ; t)$ is the Hamiltonian of the system, and summation over repeated indices is implied. Next we define a canonical transformation via the generating function of the second type according to

$S=S(\mathbf{q}, \mathbf{P} ; t)$

and assume that the Hessian matrix

$\widehat{\mathcal{H}}_{i j}(\mathbf{q}, \mathbf{P} ; t)=\frac{\partial^{2} S}{\partial q_{i} \partial P_{j}}$

of the generating function $S(\mathbf{q}, \mathbf{P} ; t)$ is non-degenerate, i.e.,

$\operatorname{det}\left(\widehat{\mathcal{H}}_{i j}\right) \neq 0$.

This implies that the inverse matrix $\widehat{\mathcal{H}}_{i j}^{-1}$ exists. The new canonical variables $(\mathbf{Q}, \mathbf{P})$ are defined by the canonical transformation as

$p_{i}=\frac{\partial S}{\partial q_{i}}, \quad Q_{i}=\frac{\partial S}{\partial P_{i}}$

We also introduce the distribution function defined in terms of the new canonical coordinates $(\mathbf{Q}, \mathbf{P})$ and the mixed pair of canonical variables $(\mathbf{q}, \mathbf{P})$ according to

$f_{0}(\mathbf{Q}, \mathbf{P} ; t)=f(\mathbf{q}(\mathbf{Q}, \mathbf{P} ; t), \mathbf{p}(\mathbf{Q}, \mathbf{P} ; t) ; t)$,

$F_{0}(\mathbf{q}, \mathbf{P} ; t)=f(\mathbf{q}, \mathbf{p}(\mathbf{q}, \mathbf{P} ; t) ; t)$

In particular, in Eq. (8) the old canonical variables are expressed in terms of the new ones, which is ensured by the implicit function theorem, provided the relation (6) holds. As 
far as the function $F_{0}(\mathbf{q}, \mathbf{P} ; t)$ is concerned, we simply replace the old momentum $\mathbf{p}$ by its counterpart taken from the first of Eqs. (7). Because

$\frac{\partial p_{i}}{\partial P_{j}}=\frac{\partial^{2} S}{\partial q_{i} \partial P_{j}}=\widehat{\mathcal{H}}_{i j} \quad \Longrightarrow \quad \frac{\partial P_{i}}{\partial p_{j}}=\widehat{\mathcal{H}}_{i j}^{-1}$,

we can express the Poisson bracket in terms of the mixed variables in the form

$[F, G]_{\mathbf{q}, \mathbf{P}}=\widehat{\mathcal{H}}_{j i}^{-1}\left(\frac{\partial F}{\partial q_{i}} \frac{\partial G}{\partial P_{j}}-\frac{\partial F}{\partial P_{j}} \frac{\partial G}{\partial q_{i}}\right)$

Differentiation of Eq. (7) with respect to time $t$, keeping the old variables (q, p) fixed, yields

$$
\begin{aligned}
& \frac{\partial^{2} S}{\partial q_{i} \partial t}+\frac{\partial^{2} S}{\partial q_{i} \partial P_{j}}\left(\frac{\partial P_{j}}{\partial t}\right)_{q p}=0 \\
& \left(\frac{\partial Q_{i}}{\partial t}\right)_{q p}=\frac{\partial^{2} S}{\partial P_{i} \partial t}+\frac{\partial^{2} S}{\partial P_{i} \partial P_{j}}\left(\frac{\partial P_{j}}{\partial t}\right)_{q p},
\end{aligned}
$$

or

$$
\left(\frac{\partial P_{j}}{\partial t}\right)_{q p}=-\widehat{\mathcal{H}}_{j i}^{-1} \frac{\partial^{2} S}{\partial q_{i} \partial t} .
$$

Our goal is to express the Vlasov equation (2) in terms of the mixed variables $(\mathbf{q}, \mathbf{P})$. Taking into account the identities

$\frac{\partial Q_{i}}{\partial q_{j}}=\frac{\partial^{2} S}{\partial q_{j} \partial P_{i}}=\widehat{\mathcal{H}}_{j i} \Longrightarrow \frac{\partial q_{i}}{\partial Q_{j}}=\widehat{\mathcal{H}}_{j i}^{-1}$

$\frac{\partial f_{0}}{\partial Q_{i}}=\widehat{\mathcal{H}}_{i j}^{-1} \frac{\partial F_{0}}{\partial q_{j}}$

$\frac{\partial f_{0}}{\partial P_{i}}=\frac{\partial F_{0}}{\partial P_{i}}-\frac{\partial f_{0}}{\partial Q_{j}} \frac{\partial^{2} S}{\partial P_{i} \partial P_{j}}$

we obtain

$$
\begin{aligned}
& \left(\frac{\partial f}{\partial t}\right)_{q p}=\frac{\partial f_{0}}{\partial t}+\frac{\partial f_{0}}{\partial Q_{i}}\left(\frac{\partial Q_{i}}{\partial t}\right)_{q p}+\frac{\partial f_{0}}{\partial P_{i}}\left(\frac{\partial P_{i}}{\partial t}\right)_{q p} \\
& =\frac{\partial F_{0}}{\partial t}+\widehat{\mathcal{H}}_{j i}^{-1}\left(\frac{\partial F_{0}}{\partial q_{i}} \frac{\partial^{2} S}{\partial t \partial P_{j}}-\frac{\partial F_{0}}{\partial P_{j}} \frac{\partial^{2} S}{\partial t \partial q_{i}}\right)
\end{aligned}
$$




$$
=\frac{\partial F_{0}}{\partial t}+\left[F_{0}, \frac{\partial S}{\partial t}\right]_{\mathbf{q}, \mathbf{P}} .
$$

Furthermore, using the relation

$[f, H]_{\mathbf{q}, \mathbf{p}}=\left[F_{0}, \mathcal{H}\right]_{\mathbf{q}, \mathbf{P}}$

where

$\mathcal{H}(\mathbf{q}, \mathbf{P} ; t)=H\left(\mathbf{q}, \nabla_{q} S ; t\right)$

we express the Vlasov equation in terms of the mixed variables according to

$\frac{\partial F_{0}}{\partial t}+\left[F_{0}, \mathcal{K}\right]_{\mathbf{q}, \mathbf{P}}=0$

where

$\mathcal{K}(\mathbf{q}, \mathbf{P} ; t)=\frac{\partial S}{\partial t}+H\left(\mathbf{q}, \nabla_{q} S ; t\right)$

is the new Hamiltonian.

For the distribution function $f_{0}(\mathbf{Q}, \mathbf{P} ; t)$, depending on the new canonical variables, we clearly obtain

$\frac{\partial f_{0}}{\partial t}+\left[f_{0}, \mathcal{K}\right]_{\mathbf{Q}, \mathbf{P}}=0$

where the new Hamiltonian $\mathcal{K}$ is a function of the new canonical pair $(\mathbf{Q}, \mathbf{P})$, such that

$\mathcal{K}\left(\nabla_{P} S, \mathbf{P} ; t\right)=\frac{\partial S}{\partial t}+H\left(\mathbf{q}, \nabla_{q} S ; t\right)$

and the Poisson bracket entering Eq. (23) has the same form as Eq. (3), expressed in the new canonical variables.

\section{PROPAGATION OF AN INTENSE BEAM THROUGH A PERIODIC FOCUS- ING LATTICE}

As a first application of the Hamiltonian formalism, we consider the propagation of a continuous beam through a periodic focusing lattice in a circular ring with radius $R$. Particle 
motion is accomplished in two degrees of freedom in a plane transverse to the design orbit. The model equations consist of the nonlinear Vlasov-Poisson equations ${ }^{1}$

$\frac{\partial f}{\partial \theta}+[f, H]_{\mathbf{q}, \mathbf{p}}=0$

$\nabla_{\mathbf{q}}^{2} \psi=-4 \pi \varrho=-4 \pi \int d^{2} \mathbf{p} f(\mathbf{q}, \mathbf{p} ; \theta)$

where

$H(\mathbf{q}, \mathbf{p} ; \theta)=\frac{R}{2}\left(p_{x}^{2}+p_{z}^{2}\right)+\frac{1}{2 R}\left(G_{x} x^{2}+G_{z} z^{2}\right)+\lambda \psi(\mathbf{q} ; \theta)$

is the normalized Hamiltonian, and $\mathbf{q}=(x, z)$. The transverse canonical momenta $\mathbf{p}=$ $\left(p_{x}, p_{z}\right)$ entering the Hamiltonian $(27)$ are dimensionless variables which represent the actual transverse momenta of the moving particle scaled by the longitudinal momentum of the synchronous particle ${ }^{10}$.

In addition, $R$ is the mean radius of the accelerator and $\psi$ is a normalized potential related to the actual electric potential $\varphi$ according to

$\psi=\frac{4 \pi \varepsilon_{0}}{N e_{b}} \varphi$

where $N$ is the total number of particles in the beam, $e_{b}$ is the particle charge, and $\varepsilon_{0}$ is the electric susceptibility of vacuum. Moreover, the parameter $\lambda$ is defined by

$\lambda=\frac{N R r_{b}}{\beta_{s}^{2} \gamma_{s}^{3}}$

where $\beta_{s}=v_{s} / c$ is the relative velocity of the synchronous particle, $\gamma_{s}=\left(1-\beta_{s}^{2}\right)^{-1 / 2}$ is the Lorentz factor, and

$r_{b}=\frac{e_{b}^{2}}{4 \pi \varepsilon_{0} m_{b} c^{2}}$

is the classical radius of a beam particle with charge $e_{b}$ and rest mass $m_{b}$. The coefficients $G_{x, z}(\theta)$ determining the focusing strength in both transverse directions are periodic functions of $\theta$ 
$G_{x, z}(\theta+\Theta)=G_{x, z}(\theta)$,

with period $\Theta$.

Following the procedure outlined in the preceding section we transform Eqs. (25) - (27) according to

$\left[F_{0}, \mathcal{K}\right]_{\mathbf{q}, \mathbf{P}} \equiv 0$

$\frac{\partial S}{\partial \theta}+\epsilon H\left(\mathbf{q}, \nabla_{\mathbf{q}} S ; \theta\right)=\mathcal{K}(\mathbf{q}, \mathbf{P})$,

$\nabla_{\mathbf{q}}^{2} \psi=-4 \pi \int d^{2} \mathbf{P} F_{0}(\mathbf{q}, \mathbf{P}) \operatorname{det}\left(\nabla_{\mathbf{q}} \nabla_{\mathbf{P}} S\right)$,

where $\epsilon$ is formally a small parameter proportional to the focusing field strength, which will be set equal to unity at the end of the calculation. The next step is to expand the quantities $S, \mathcal{K}$ and $\psi$ in a power series in $\epsilon$ according to

$S=S_{0}+\epsilon S_{1}+\epsilon^{2} S_{2}+\epsilon^{3} S_{3}+\ldots$,

$\mathcal{K}=\mathcal{K}_{0}+\epsilon \mathcal{K}_{1}+\epsilon^{2} \mathcal{K}_{2}+\epsilon^{3} \mathcal{K}_{3}+\ldots$,

$\psi=\psi_{0}+\epsilon \psi_{1}+\epsilon^{2} \psi_{2}+\epsilon^{3} \psi_{3}+\ldots$

We now substitute the expansions (35) - (37) into Eqs. (33) and (34) and obtain perturbation equations that can be solved order by order.

The lowest order solution is evident and has the form

$S_{0}=\mathbf{q} \cdot \mathbf{P}, \quad \mathcal{K}_{0} \equiv 0$,

$\nabla_{\mathbf{q}}^{2} \psi_{0}=-4 \pi \int d^{2} \mathbf{P} F_{0}(\mathbf{q}, \mathbf{P})$.

First order $O(\epsilon)$ : Taking into account the already obtained lowest order solutions (38) and (39), the Hamilton-Jacobi equation (33) to first order in $\epsilon$ can be expressed as 
$\frac{\partial S_{1}}{\partial \theta}+\frac{R}{2}\left(P_{x}^{2}+P_{z}^{2}\right)+\frac{1}{2 R}\left(G_{x} x^{2}+G_{z} z^{2}\right)+\lambda \psi_{0}=\mathcal{K}_{1}(\mathbf{q}, \mathbf{P})$

Imposing the condition that the first order Hamiltonian $\mathcal{K}_{1}$ be equal to

$\mathcal{K}_{1}(\mathbf{q}, \mathbf{P})=\frac{R}{2}\left(P_{x}^{2}+P_{z}^{2}\right)+\frac{1}{2 R}\left(\bar{G}_{x} x^{2}+\bar{G}_{z} z^{2}\right)+\lambda \psi_{0}(\mathbf{q})$,

we obtain immediately

$S_{1}=-\frac{1}{2 R}\left[\widetilde{G}_{x}(\theta) x^{2}+\widetilde{G}_{z}(\theta) z^{2}\right]$

$\psi_{1} \equiv 0$.

Here we have introduced the notation

$\bar{G}_{x, z}=\frac{1}{\Theta} \int_{\theta_{0}}^{\theta_{0}+\Theta} d \theta G_{x, z}(\theta), \quad \widetilde{G}_{x, z}(\theta)=\int_{\theta_{0}}^{\theta_{0}+\theta} d \tau\left[G_{x, z}(\tau)-\bar{G}_{x, z}\right]$.

Note that since the focusing coefficients are periodic functions of $\theta$ they can be expanded in a Fourier series

$G_{x, z}(\theta)=\sum_{n=-\infty}^{\infty} G_{x, z}^{(n)} \exp (i n \Omega \theta)$

where

$G_{x, z}^{(n)}=\frac{1}{\Theta} \int_{0}^{\Theta} d \theta G_{x, z}(\theta) \exp (-i n \Omega \theta)$

and $\Omega=2 \pi / \Theta$. Therefore for the quantities $\bar{G}_{x, z}$ and $\widetilde{G}_{x, z}(\theta)$ expressed in terms of the Fourier amplitudes, we obtain

$\bar{G}_{x, z}=G_{x, z}^{(0)}, \quad \widetilde{G}_{x, z}(\theta)=-\frac{i}{\Omega} \sum_{n \neq 0} \frac{G_{x, z}^{(n)}}{n} \exp (i n \Omega \theta)$.

Second order $O\left(\epsilon^{2}\right)$ : To this order, the Hamilton-Jacobi equation (33) takes the form

$\frac{\partial S_{2}}{\partial \theta}-\left(x P_{x} \widetilde{G}_{x}+z P_{z} \widetilde{G}_{z}\right)=\mathcal{K}_{2}(\mathbf{q}, \mathbf{P})$

It is straightforward to solve Eq. (48), yielding the obvious result 
$S_{2}=x P_{x} \widetilde{\widetilde{G}}_{x}(\theta)+z P_{z} \widetilde{\widetilde{G}}_{z}(\theta), \quad \mathcal{K}_{2}(\mathbf{q}, \mathbf{P}) \equiv 0$

For the second order potential $\psi_{2}$ we obtain the equation

$\nabla_{\mathbf{q}}^{2} \psi_{2}=-4 \pi\left(\widetilde{\widetilde{G}}_{x}+\widetilde{\widetilde{G}}_{z}\right) \int d^{2} \mathbf{P} F_{0}(\mathbf{q}, \mathbf{P})$,

or, making use of (39),

$\psi_{2}(\mathbf{q} ; \theta)=\left[\widetilde{\widetilde{G}}_{x}(\theta)+\widetilde{\widetilde{G}}_{z}(\theta)\right] \psi_{0}(\mathbf{q})$

In Eqs. $(49)-(51), \widetilde{\widetilde{G}}_{x, z}(\theta)$ denotes application of the integral operation in Eq. (44) to $\widetilde{G}_{x, z}(\theta)$, i.e.,

$\widetilde{\widetilde{G}}_{x, z}(\theta)=\int_{\theta_{0}}^{\theta_{0}+\theta} d \tau \widetilde{G}_{x, z}(\tau)$

because $\widetilde{\widetilde{G}}_{x, z}=0$.

Third order $O\left(\epsilon^{3}\right)$ : To third order in $\epsilon$, the Hamilton-Jacobi equation (33) can be written as

$\frac{\partial S_{3}}{\partial \theta}+R\left(P_{x}^{2} \widetilde{\widetilde{G}}_{x}+P_{z}^{2} \widetilde{\widetilde{G}}_{z}\right)+\frac{1}{2 R}\left(\widetilde{G}_{x}^{2} x^{2}+\widetilde{G}_{z}^{2} z^{2}\right)+\lambda\left(\widetilde{\widetilde{G}}_{x}+\widetilde{\widetilde{G}}_{z}\right) \psi_{0}=\mathcal{K}_{3}(\mathbf{q}, \mathbf{P})$

The third-order Hamiltonian $\mathcal{K}_{3}$ is given by the expression

$\mathcal{K}_{3}(\mathbf{q}, \mathbf{P})=\frac{1}{2 R}\left(\overline{\widetilde{G}_{x}^{2}} x^{2}+\overline{\widetilde{G}_{z}^{2}} z^{2}\right)$

Equation (53) can be easily solved for the third-order generating function $S_{3}$. The result is

$S_{3}=-R\left(P_{x}^{2} \widetilde{\widetilde{G}}_{x}+P_{z}^{2} \widetilde{\widetilde{G}}_{z}\right)-\frac{1}{2 R}\left(\widetilde{\widetilde{G}}_{x}^{2} x^{2}+\widetilde{\widetilde{G}}_{z}^{2} z^{2}\right)-\lambda\left(\widetilde{\widetilde{G}}_{x}+\widetilde{\widetilde{G}}_{z}\right) \psi_{0}$.

For the third-order electric potential $\psi_{3}$ we obtain simply

$\psi_{3} \equiv 0$

Fourth order $O\left(\epsilon^{4}\right)$ : To the fourth order in the expansion parameter $\epsilon$ the HamiltonJacobi equation (33) can be expressed as 
$\frac{\partial S_{4}}{\partial \theta}-x P_{x}\left(\widetilde{\widetilde{G}_{x}^{2}}+\widetilde{G}_{x} \widetilde{\widetilde{G}}_{x}\right)-z P_{z}\left(\widetilde{\widetilde{G}}_{z}^{2}+\widetilde{G}_{z} \widetilde{\widetilde{G}}_{z}\right)-\lambda R\left(\widetilde{\widetilde{G}}_{x}+\widetilde{\widetilde{G}}_{z}\right)\left(P_{x} \frac{\partial \psi_{0}}{\partial x}+P_{z} \frac{\partial \psi_{0}}{\partial z}\right)=\mathcal{K}_{4}(\mathbf{q}, \mathbf{P})$

The obvious condition to impose is that the fourth-order Hamiltonian $\mathcal{K}_{4}$ be equal to

$\mathcal{K}_{4}(\mathbf{q}, \mathbf{P})=-x P_{x} \overline{\widetilde{G}_{x} \widetilde{\widetilde{G}}_{x}}-z P_{z} \overline{\widetilde{G}_{z} \widetilde{\widetilde{G}}_{z}}$

With Eq. (58) in hand, it is straightforward to solve the fourth-order Hamilton-Jacobi equation (57) for $S_{4}$. We obtain

$S_{4}=x P_{x}\left(\widetilde{\widetilde{\widetilde{G}_{x}^{2}}}+\widetilde{\widetilde{G}_{x} \widetilde{\widetilde{G}}_{x}}\right)+z P_{z}\left(\widetilde{\widetilde{\widetilde{G}}_{z}^{2}}+\widetilde{\widetilde{G}_{z} \widetilde{\widetilde{G}}_{z}}\right)+\lambda R\left(\widetilde{\widetilde{G}}_{x}+\widetilde{\widetilde{G}}_{z}\right)\left(P_{x} \frac{\partial \psi_{0}}{\partial x}+P_{z} \frac{\partial \psi_{0}}{\partial z}\right)$.

For the fourth-order electric potential $\psi_{4}$, we obtain the Poisson equation

$\nabla_{\mathbf{q}}^{2} \psi_{4}=\left[\widetilde{\widetilde{G}}_{x} \widetilde{\widetilde{G}}_{z}+\widetilde{\widetilde{\widetilde{G}}_{x}^{2}}+\widetilde{\widetilde{G}}_{z}^{2}+\widetilde{G}_{x} \widetilde{\widetilde{G}}_{x}+\widetilde{\widetilde{G}_{z}} \widetilde{\widetilde{G}}_{z}+\lambda R\left(\widetilde{\widetilde{G}}_{x}+\widetilde{\widetilde{G}}_{z}\right) \nabla_{\mathbf{q}}^{2} \psi_{0}\right] \nabla_{\mathbf{q}}^{2} \psi_{0}$.

Fifth order $O\left(\epsilon^{5}\right)$ : In fifth order, we are interested in the Hamiltonian $\mathcal{K}_{5}$. Omitting algebraic details we find

$$
\begin{aligned}
& \mathcal{K}_{5}(\mathbf{q}, \mathbf{P})=\frac{R}{2}\left(\overline{\widetilde{\widetilde{G}}_{x}^{2}} P_{x}^{2}+\overline{\widetilde{\widetilde{G}}_{z}^{2}} P_{z}^{2}\right)+\frac{1}{R}\left(\overline{\widetilde{G}_{x} \widetilde{\widetilde{G}}_{x}^{2}} x^{2}+\overline{\widetilde{G}_{z} \widetilde{\widetilde{G}}_{z}^{2}} z^{2}\right) \\
& \left.+\lambda \overline{\widetilde{G}_{x}\left(\widetilde{\widetilde{G}}_{x}+\widetilde{\widetilde{G}}_{z}\right)} x \frac{\partial \psi_{0}}{\partial x}+\overline{\widetilde{G}_{z}\left(\widetilde{\widetilde{G}}_{x}+\widetilde{\widetilde{G}}_{z}\right)} z \frac{\partial \psi_{0}}{\partial z}\right] .
\end{aligned}
$$

In concluding this section, we collect terms up to fifth order in $\epsilon$ in the new Hamiltonian $\mathcal{K}=\mathcal{K}_{0}+\epsilon \mathcal{K}_{1}+\epsilon^{2} \mathcal{K}_{2}+\ldots$ and set $\epsilon=1$. This gives

$$
\begin{aligned}
& \mathcal{K}(\mathbf{q}, \mathbf{P})=\sum_{u=(x, z)}\left(\frac{R \mathcal{A}_{u}}{2} P_{u}^{2}+\mathcal{B}_{u} u P_{u}+\frac{\mathcal{C}_{u}}{2 R} u^{2}\right)+\lambda \psi_{0}(\mathbf{q}) \\
& \left.+\lambda \overline{\widetilde{G}_{x}\left(\widetilde{\widetilde{G}}_{x}+\widetilde{\widetilde{G}}_{z}\right)} x \frac{\partial \psi_{0}}{\partial x}+\overline{\widetilde{G}_{z}\left(\widetilde{\widetilde{G}}_{x}+\widetilde{\widetilde{G}}_{z}\right)} z \frac{\partial \psi_{0}}{\partial z}\right]
\end{aligned}
$$

where the coefficients $\mathcal{A}_{u}, \mathcal{B}_{u}$ and $\mathcal{C}_{u}$ are defined by the expressions

$\mathcal{A}_{u}=1+\epsilon^{4} \overline{\widetilde{G}}_{u}^{2}$ 
$\mathcal{B}_{u}=-\epsilon^{3} \overline{\widetilde{G}_{u} \widetilde{G}_{u}}$,

$\mathcal{C}_{u}=\bar{G}_{u}+\epsilon^{2} \overline{\widetilde{G}_{u}^{2}}+2 \epsilon^{4} \overline{\widetilde{G}_{u} \widetilde{\widetilde{G}_{u}^{2}}}$

The Hamiltonian (62), neglecting the contribution from the self-field $\psi_{0}$, describes the unperturbed betatron oscillations in both horizontal and vertical directions.

It is useful to compute the unperturbed betatron tunes $\nu_{x, z}$ in terms of averages over the focusing field-strengths. For a Hamiltonian system governed by a quadratic form in the canonical variables of the type in Eq. (62), it is well-known that the characteristic frequencies $\nu_{x, z}$ can be expressed as

$\nu_{u}^{2}=\mathcal{A}_{u} \mathcal{C}_{u}-\mathcal{B}_{u}^{2}, \quad(u=x, z)$

Keeping terms up to sixth order in the perturbation parameter $\epsilon$, we obtain

$\nu_{u}^{2}=\bar{G}_{u}+\epsilon^{2}{\widetilde{\widetilde{G}_{u}^{2}}}^{2}+\epsilon^{4}\left(\bar{G}_{u} \overline{\widetilde{\widetilde{G}}_{u}^{2}}+2 \overline{\widetilde{G}_{u} \widetilde{\widetilde{G}}_{u}^{2}}\right)+\epsilon^{6}\left[\overline{\widetilde{G}_{u}^{2}} \overline{\widetilde{\widetilde{G}}_{u}^{2}}-\left(\overline{\widetilde{G}_{u} \widetilde{\widetilde{G}}_{u}}\right)^{2}\right]$.

In terms of Fourier amplitudes of the focusing coefficients, the Eq. (67) can be expressed as

$$
\begin{aligned}
& \nu_{u}^{2}=G_{u}^{(0)}+\frac{2 \epsilon^{2}}{\Omega^{2}} \sum_{n=1}^{\infty} \frac{\left|G_{u}^{(n)}\right|^{2}}{n^{2}}+\frac{2 \epsilon^{4}}{\Omega^{4}}\left[G_{u}^{(0)} \sum_{n=1}^{\infty} \frac{\left|G_{u}^{(n)}\right|^{2}}{n^{4}}+2 \sum_{\substack{m, n=1 \\
m \neq n}}^{\infty} \frac{\operatorname{Re}\left(G_{u}^{(m) *} G_{u}^{(n)} G_{u}^{(m-n)}\right)}{m n(m-n)^{2}}\right. \\
& \left.-2 \sum_{m, n=1}^{\infty} \frac{\operatorname{Re}\left(G_{u}^{(m)} G_{u}^{(n)} G_{u}^{(m+n) *}\right)}{m n(m+n)^{2}}\right]+\frac{4 \epsilon^{6}}{\Omega^{6}} \sum_{m, n=1}^{\infty} \frac{\left|G_{u}^{(m)}\right|^{2}\left|G_{u}^{(n)}\right|^{2}}{m^{2} n^{4}} .
\end{aligned}
$$

For purposes of illustration, we consider a simple FODO lattice with equal focusing and defocusing strengths $+G$ and $-G$, and period $\Theta$. We also assume that the longitudinal dimensions $\theta_{f}$ of the focusing and defocusing lenses are equal; the longitudinal dimensions $\theta_{d}$ of the corresponding drift spaces are assumed to be equal as well. Moreover,

$2\left(\theta_{f}+\theta_{d}\right)=\Theta$.

For simplicity we consider the horizontal degree of freedom only (the vertical one can be treated in analogous manner). The Fourier amplitudes of the focusing coefficients are 
$G_{x}^{(2 n+1)}=\frac{i G}{(2 n+1) \pi}\left\{\exp \left[-i(2 n+1) \Omega \theta_{f}\right]-1\right\}, \quad G_{x}^{(2 n)}=0$,

where $n=0,1,2, \ldots$. To second order in $\epsilon$, we obtain for the horizontal betatron tune

$\nu_{x}^{2}=\frac{2 \epsilon^{2} \Theta^{2} G^{2}}{\pi^{4}} \sum_{m=1}^{\infty} \frac{1}{(2 m-1)^{4}} \sin ^{2} \frac{(2 m-1) \pi \theta_{f}}{\Theta}$.

In the limit of infinitely thin lenses, $\theta_{f} \rightarrow 0$, Eq. (71) reduces to the well-known expression

$\nu_{x}^{2}=\frac{\epsilon^{2} \theta_{f}^{2} G^{2}}{4}$

where use of the identity

$\sum_{m=1}^{\infty} \frac{1}{(2 m-1)^{2}}=\frac{\pi^{2}}{8}$

has been made.

It is evident from Eqs. (68) and (71), that the Hamiltonian averaging technique developed here represents a powerful formalism for evaluating the betatron tunes in terms of averages over the focusing field strength.

\section{COHERENT BEAM-BEAM INTERACTION}

As a second application of the Hamiltonian formalism developed in Sec. 2, we study here the evolution of two counter-propagating beams, nonlinearly coupled by the electromagnetic interaction between the beams at collision. For simplicity, we consider one-dimensional motion in the vertical $(q)$ direction, described by the nonlinear Vlasov-Poisson equations

$\frac{\partial f_{k}}{\partial \theta}+\left[f_{k}, H_{k}\right]=0$

$\frac{\partial^{2} V_{k}}{\partial q^{2}}=4 \pi \int d p f_{3-k}(q, p ; \theta)$

where

$H_{k}=\frac{\nu_{k}}{2}\left(p^{2}+q^{2}\right)+\lambda_{k} \delta_{p}(\theta) V_{k}(q ; \theta)$ 
is the Hamiltonian. Here $\lambda_{k}$ is the beam-beam coupling parameter, defined according to ${ }^{11}$

$\lambda_{k}=\frac{R r_{e} N_{3-k} \beta_{k q}^{*}}{\gamma_{k 0} L_{(3-k) x}} \frac{1+\beta_{k 0} \beta_{(3-k) 0}}{\beta_{k 0}^{2}} \approx \frac{2 R r_{e} N_{3-k} \beta_{k q}^{*}}{\gamma_{k 0} L_{(3-k) x}}$.

Moreover, $(k=1,2)$ labels the beam, $f_{k}(q, p ; \theta)$ is the distribution function, $\theta$ is the azimuthal angle, and $\nu_{k}$ is the betatron frequency in vertical direction. In addition, $R$ is the mean machine radius, $r_{e}$ is the classical electron radius, $N_{1,2}$ is the total number of particles in either beam, $V_{k}(q ; \theta)$ is the normalized beam-beam potential, $\beta_{k q}^{*}$ is the vertical beta-function at the interaction point, and $L_{k x}$ is the horizontal dimension of the beam ribbon $^{12}$.

Our goal is to determine a canonical transformation such that the new Hamiltonian is time-independent. As a consequence, the stationary solution of the Vlasov equation (21) is expressed as a function of the new Hamiltonian. Following the procedure outlined in the Sec. 2 we transform Eqs. (74) - (76) according to

$$
\begin{aligned}
& {\left[F_{0}^{(k)}, \mathcal{K}_{k}\right] \equiv 0,} \\
& \frac{\partial S_{k}}{\partial \theta}+\epsilon H_{k}\left(q, \frac{\partial S_{k}}{\partial q} ; \theta\right)=\mathcal{K}_{k}(q, P), \\
& \frac{\partial^{2} V_{k}}{\partial q^{2}}=4 \pi \int d P \frac{\partial^{2} S_{k}}{\partial q \partial P} F_{0}^{(3-k)}(q, P),
\end{aligned}
$$

where $\epsilon$ is again a formal small parameter, which will be set equal to unity at the end of the calculation.

The next step is to expand the quantities $S_{k}, \mathcal{K}_{k}$ and $V_{k}$ in a power series in $\epsilon$, analogous to Eqs. (35) - (37), according to

$$
\begin{aligned}
& S_{k}=q P+\epsilon G_{k}^{(1)}+\epsilon^{2} G_{k}^{(2)}+\epsilon^{3} G_{k}^{(3)}+\ldots, \\
& \mathcal{K}_{k}=\epsilon \mathcal{K}_{k}^{(1)}+\epsilon^{2} \mathcal{K}_{k}^{(2)}+\epsilon^{3} \mathcal{K}_{k}^{(3)}+\ldots, \\
& V_{k}=\widetilde{V}_{k}+\epsilon V_{k}^{(1)}+\epsilon^{2} V_{k}^{(2)}+\epsilon^{3} V_{k}^{(3)}+\ldots,
\end{aligned}
$$


where

$\frac{\partial^{2} \widetilde{V}_{k}}{\partial q^{2}}=4 \pi \int d P F_{0}^{(3-k)}(q, P)$.

Substitution of the above expansions (81) - (83) into Eqs. (79) and (80) yields perturbation equations that can be solved successively order by order. The results to third order in $\epsilon$ are briefly summarized below.

First Order: $O(\epsilon)$

$\mathcal{K}_{k}^{(1)}(q, P)=\frac{\nu_{k}}{2}\left(P^{2}+q^{2}\right)+\frac{\lambda_{k}}{2 \pi} \widetilde{V}_{k}(q)$,

$G_{k}^{(1)}(q, P ; \theta)=\frac{i \lambda_{k}}{2 \pi} \widetilde{V}_{k}(q) \sum_{n \neq 0} \frac{1}{n} \exp (i n \theta)$,

$V_{k}^{(1)}(q ; \theta) \equiv 0$.

Second Order: $O\left(\epsilon^{2}\right)$

$\mathcal{K}_{k}^{(2)}(q, P) \equiv 0$

$G_{k}^{(2)}(q, P ; \theta)=-\frac{\lambda_{k} \nu_{k}}{2 \pi} P \tilde{V}_{k}^{\prime}(q) \sum_{n \neq 0} \frac{1}{n^{2}} \exp (i n \theta)$,

$V_{k}^{(2)}(q ; \theta)=-\frac{\lambda_{k} \nu_{k}}{2 \pi} \widetilde{V}_{k}^{(2)}(q) \sum_{n \neq 0} \frac{1}{n^{2}} \exp (i n \theta)$

where

$\frac{\partial^{2} \widetilde{V}_{k}^{(2)}}{\partial q^{2}}=4 \pi \widetilde{V}_{k}^{\prime \prime}(q) \int d P F_{0}^{(3-k)}(q, P)$.

Third Order: $O\left(\epsilon^{3}\right)$ In third order we are interested in the new Hamiltonian, which is of the form

$\mathcal{K}_{k}^{(3)}(q, P)=\frac{\lambda_{k}^{2} \nu_{k}}{4 \pi^{2}} \zeta(2)\left[\widetilde{V}_{k}^{\prime 2}(q)-2 \widetilde{V}_{k}^{(2)}(q)\right]$

where $\zeta(z)$ is Riemann's zeta-function ${ }^{13}$ 
$\zeta(z)=\sum_{n=1}^{\infty} \frac{1}{n^{z}}$

The effectiveness of the Hamiltonian formalism developed in the present paper has been demonstrated in two particular examples. In the first example discussed in the previous section, the short-scale dynamics is contained in the external focusing force acting on the beam, while an essential feature of the coherent beam-beam interaction treated above is the relatively fast variation of the collective interaction between the two colliding beams. The simplicity in applying the Hamiltonian averaging technique is embedded in the use of mixed canonical variables. Instead of expanding the generating function and the new Hamiltonian in terms of the new canonical coordinates and momenta ${ }^{7,8}$ one has to simply solve the Hamilton-Jacobi equations order by order. It should be pointed out that the mixed variable Hamiltonian formalism can be used to derive amplitude equations, describing processes of formation of patterns and coherent structures in a number of plasma and beam systems in which collective processes are important.

\section{THE EQUILIBRIUM DISTRIBUTION FUNCTION}

Since the new Hamiltonian $\mathcal{K}_{k}$ is time-independent (by construction), the equilibrium distribution function $F_{0}^{(k)}$ [see Eq. (78)] is a function of the new Hamiltonian

$F_{0}^{(k)}(q, P)=\mathcal{G}_{k}\left(\mathcal{K}_{k}\right)$

where

$\mathcal{K}_{k}(q, P)=\frac{\nu_{k}}{2}\left(P^{2}+q^{2}\right)+\frac{\lambda_{k}}{2 \pi} \widetilde{V}_{k}(q)+\frac{\lambda_{k}^{2} \nu_{k}}{4 \pi^{2}} \zeta(2)\left[\widetilde{V}_{k}^{\prime 2}(q)-2 \widetilde{V}_{k}^{(2)}(q)\right]$

Integrating Eq. (94) over $P$ we obtain a nonlinear integral equation of the Haissinski type ${ }^{14}$

for the equilibrium beam density profile $\varrho_{0}^{(k)}$

$\varrho_{0}^{(k)}(q)=\int d P \mathcal{G}_{k}\left(\mathcal{K}_{k}\right)$

where 
$\mathcal{K}_{k}(q, P)=\frac{\nu_{k}}{2}\left(P^{2}+q^{2}\right)+\lambda_{k} \int d q^{\prime}\left|q-q^{\prime}\right| \varrho_{0}^{(3-k)}\left(q^{\prime}\right)+2 \lambda_{k}^{2} \nu_{k} \zeta(2) \mathcal{F}_{k}(q)$,

$\mathcal{F}_{k}(q)=\int d q^{\prime} d q^{\prime \prime} \mathcal{Z}\left(q-q^{\prime}, q^{\prime}-q^{\prime \prime}\right) \varrho_{0}^{(3-k)}\left(q^{\prime}\right) \varrho_{0}^{(3-k)}\left(q^{\prime \prime}\right)$,

$\mathcal{Z}(u, v)=\operatorname{sgn}(u) \operatorname{sgn}(v)-2|u| \delta(v)$.

Here $\operatorname{sgn}(z)$ is the well-known sign-function.

Let us further specify the function $\mathcal{G}_{k}\left(\mathcal{K}_{k}\right)$ and assume that it is given by the thermal equilibrium distribution ${ }^{1,10,15}$

$\mathcal{G}_{k}\left(\mathcal{K}_{k}\right)=\mathcal{N}_{k} \exp \left(-\frac{\mathcal{K}_{k}}{\varepsilon_{k}}\right)$,

where $\mathcal{N}_{k}$ is a normalization constant, defined according to

$\frac{1}{\mathcal{N}_{k}}=\int d q d P \exp \left[-\frac{\mathcal{K}_{k}(q, P)}{\varepsilon_{k}}\right]$

and $\varepsilon_{k}$ is the unnormalized beam emittance. The second term in the Hamiltonian (97) can be transformed according to

$$
\begin{aligned}
& \int_{-\infty}^{\infty} d q^{\prime}\left|q-q^{\prime}\right| \varrho_{0}^{(3-k)}\left(q^{\prime}\right)=q-\left\langle q_{3-k}\right\rangle+2 \int_{q}^{\infty} d q^{\prime}\left(q^{\prime}-q\right) \varrho_{0}^{(3-k)}\left(q^{\prime}\right) \\
& =q-\left\langle q_{3-k}\right\rangle+2 \int_{0}^{\infty} d q_{1} q_{1} \varrho_{0}^{(3-k)}\left(q_{1}+q\right),
\end{aligned}
$$

where

$$
\left\langle q_{k}^{n}\right\rangle=\int_{-\infty}^{\infty} d q q^{n} \varrho_{0}^{(k)}(q)
$$

Expanding the beam density $\varrho_{0}^{(3-k)}\left(q_{1}+q\right)$ occurring in the integral in Eq. (102) in a Taylor series and integrating by parts, we obtain

$$
\int_{-\infty}^{\infty} d q^{\prime}\left|q-q^{\prime}\right| \varrho_{0}^{(3-k)}\left(q^{\prime}\right)=\left\langle q_{3-k}^{(+)}\right\rangle-\left\langle q_{3-k}^{(-)}\right\rangle+\left(1-2 \mathcal{A}_{3-k}\right) q+2 \sum_{n=2}^{\infty} \frac{\mathcal{C}_{3-k}^{(n)}}{n !} q^{n}
$$

where 


$$
\begin{array}{ll}
\left\langle q_{k}^{(+)}\right\rangle=\int_{0}^{\infty} d q q \varrho_{0}^{(k)}(q), & \left\langle q_{k}^{(-)}\right\rangle=\int_{-\infty}^{0} d q q \varrho_{0}^{(k)}(q), \\
\mathcal{A}_{k}=\int_{0}^{\infty} d q \varrho_{0}^{(k)}(q), & \mathcal{C}_{k}^{(n)}=\left.\frac{\partial^{n-2} \varrho_{0}^{(k)}(q)}{\partial q^{n-2}}\right|_{q=0} .
\end{array}
$$

Substituting (100) and (104) into Eq. (96) we obtain

$$
\varrho_{0}^{(k)}(q)=\mathcal{N}_{k} \sqrt{\frac{2 \pi \varepsilon_{k}}{\nu_{k}}} \exp \left[-\frac{\nu_{k} q^{2}}{2 \varepsilon_{k}}-\frac{\lambda_{k}}{\varepsilon_{k}}\left(1-2 \mathcal{A}_{3-k}\right) q-\frac{2 \lambda_{k}}{\varepsilon_{k}} \sum_{n=2}^{\infty} \frac{\mathcal{C}_{3-k}^{(n)}}{n !} q^{n}\right] .
$$

Taking into account that

$$
\mathcal{A}_{k}=\frac{\pi \varepsilon_{k}}{\nu_{k}} \mathcal{N}_{k}+O\left(\lambda_{k}\right), \quad \mathcal{C}_{k}^{(n)}=\mathcal{N}_{k} \sqrt{\frac{2 \pi \varepsilon_{k}}{\nu_{k}}}(-1)^{n-2}\left(\frac{\nu_{k}}{2 \varepsilon_{k}}\right)^{(n-2) / 2} H_{n-2}(0)+O\left(\lambda_{k}\right)
$$

where $H_{n}(z)$ is the Hermite polynomial ${ }^{13}$ of order $n$, we obtain

$\varrho_{0}^{(k)}(q)=\mathcal{N}_{k} \sqrt{\frac{2 \pi \varepsilon_{k}}{\nu_{k}}} \exp \left[h_{k}(q)\right]$

where

$h_{k}(q)=-\frac{\nu_{k} q^{2}}{2 \varepsilon_{k}}-\frac{\lambda_{k} \mathcal{B}_{3-k} q}{\varepsilon_{k}}-\frac{2 \pi \lambda_{k} \varepsilon_{3-k} \mathcal{N}_{3-k}}{\varepsilon_{k} \nu_{3-k}}\left[q \Phi\left(a_{3-k} q\right)+\frac{e^{-a_{3-k}^{2} q^{2}}}{a_{3-k} \sqrt{\pi}}\right]$,

and

$\mathcal{B}_{k}=1-\frac{2 \pi \varepsilon_{k} \mathcal{N}_{k}}{\nu_{k}}, \quad \quad a_{k}^{2}=\frac{\nu_{k}}{2 \varepsilon_{k}}$

Here, $\Phi(z)$ is the error function ${ }^{13}$.

In order to determine the normalization constant $(\mathrm{s}), \mathcal{N}_{k}$, we utilize the method of Laplace to take the integral of the beam density $\varrho_{0}^{(k)}(q)$ over $q$. The first step consists in finding the extremum value(s) $q_{k}^{(e)}$ of the function $(\mathrm{s}) h_{k}(q)$. They satisfy the (two) equation(s)

$$
\frac{\nu_{k} q_{k}^{(e)}}{\varepsilon_{k}}+\frac{\lambda_{k} \mathcal{B}_{3-k}}{\varepsilon_{k}}+\frac{2 \pi \lambda_{k} \varepsilon_{3-k} \mathcal{N}_{3-k}}{\varepsilon_{k} \nu_{3-k}} \Phi\left(a_{3-k} q_{k}^{(e)}\right)=0
$$

These are evidently maxima, since

$h_{k}^{\prime \prime}\left(q_{k}^{(e)}\right)=-\frac{\nu_{k}}{\varepsilon_{k}}-\frac{4 \pi \lambda_{k} \varepsilon_{3-k} \mathcal{N}_{3-k}}{\varepsilon_{k} \nu_{3-k}} \frac{a_{3-k}}{\sqrt{\pi}} e^{-a_{3-k}^{2} q_{k}^{(e) 2}}<0$. 
Integrating the beam density (109) over $q$, we obtain ${ }^{16}$

$1=2 \pi \mathcal{N}_{k} \sqrt{\frac{\varepsilon_{k}}{\nu_{k}\left|h_{k}^{\prime \prime}\left(q_{k}^{(e)}\right)\right|}} \exp \left[h_{k}\left(q_{k}^{(e)}\right)\right]$.

Equation (114) represents two transcendental equations for determining the normalization constants $\mathcal{N}_{k}$. For the beam centroid and the beam size, i.e., the first and the second moments of the beam density (109), we obtain

$$
\begin{aligned}
& \left\langle q_{k}\right\rangle=q_{k}^{(e)}+\frac{2 \mathcal{N}_{k}}{\left|h_{k}^{\prime \prime}\left(q_{k}^{(e)}\right)\right|} \sqrt{\frac{2 \pi \varepsilon_{k}}{\nu_{k}}} \exp \left[h_{k}\left(q_{k}^{(e)}\right)\right] \\
& \left\langle q_{k}^{2}\right\rangle=q_{k}^{(e) 2}+\frac{4 \mathcal{N}_{k} q_{k}^{(e)}}{\left|h_{k}^{\prime \prime}\left(q_{k}^{(e)}\right)\right|} \sqrt{\frac{2 \pi \varepsilon_{k}}{\nu_{k}}} \exp \left[h_{k}\left(q_{k}^{(e)}\right)\right]+2 \pi \mathcal{N}_{k} \sqrt{\frac{\varepsilon_{k}}{\nu_{k}}}\left[\frac{1}{\left|h_{k}^{\prime \prime}\left(q_{k}^{(e)}\right)\right|}\right]^{3 / 2} \exp \left[h_{k}\left(q_{k}^{(e)}\right)\right] .
\end{aligned}
$$

In order to proceed further, we assume that the beam-beam coupling parameter $\lambda_{k}$ is small, and expand the equilibrium beam density $\varrho_{0}^{(k)}(q)$ in a perturbation series in $\lambda_{k}$ according to

$\varrho_{0}^{(k)}(q)=\varrho_{00}^{(k)}(q)+\lambda_{k} \varrho_{01}^{(k)}(q)+\ldots$

where

$\varrho_{00}^{(k)}(q)=\frac{\mathcal{N}_{k} \sqrt{\pi}}{a_{k}} \exp \left(-a_{k}^{2} q^{2}\right)$

and

$\varrho_{01}^{(k)}(q)=-\frac{1}{\varepsilon_{k}}\left\{\mathcal{B}_{3-k} q+\frac{\pi \mathcal{N}_{3-k}}{a_{3-k}^{2}}\left[q \Phi\left(a_{3-k} q\right)+\frac{e^{-a_{3-k}^{2} q^{2}}}{a_{3-k} \sqrt{\pi}}\right]\right\} \varrho_{00}^{(k)}(q)$.

The main goal in what follows is to determine the normalization constant(s) $\mathcal{N}_{k 0}$. To do so we integrate Eq. (117) over $q$. As a result of simple algebraic manipulations, we obtain

$\frac{\pi \mathcal{N}_{k}}{a_{k}^{2}}-\frac{\pi \sqrt{\pi} \lambda_{k}}{\varepsilon_{k} a_{k}^{3} a_{3-k}^{3}} \sqrt{a_{k}^{2}+a_{3-k}^{2}} \mathcal{N}_{k} \mathcal{N}_{3-k}=1$

Introducing the new unknowns 
$\mathcal{M}_{k}=\frac{\pi \mathcal{N}_{k}}{a_{k}^{2}}$

we can write the two equations for determining $\mathcal{M}_{1,2}$ as

$1=\mathcal{M}_{1}-b_{1} \mathcal{M}_{1} \mathcal{M}_{2}$

$1=\mathcal{M}_{2}-b_{2} \mathcal{M}_{1} \mathcal{M}_{2}$

where

$b_{1}=\frac{\lambda_{1}}{\varepsilon_{1} \sqrt{\pi}} \frac{\sqrt{a_{1}^{2}+a_{2}^{2}}}{a_{1} a_{2}}, \quad b_{2}=\frac{\lambda_{2}}{\varepsilon_{2} \sqrt{\pi}} \frac{\sqrt{a_{1}^{2}+a_{2}^{2}}}{a_{1} a_{2}}$.

From Eq. (122), as a result of simple algebraic manipulations we obtain the quadratic equation

$b_{2} \mathcal{M}_{1}^{2}-\left(b_{2}-b_{1}+1\right) \mathcal{M}_{1}+1=0$

for $\mathcal{M}_{1}$, and the equation

$b_{1} \mathcal{M}_{2}=b_{2} \mathcal{M}_{1}+b_{1}-b_{2}$

for determining $\mathcal{M}_{2}$ once $\mathcal{M}_{1}$ is known. Equation (124) has one real double root if and only if the discriminant

$\mathcal{D}=\left(b_{2}-b_{1}+1\right)^{2}-4 b_{2}$

is equal to zero. This gives

$b_{2}=\left(\sqrt{b_{1}} \pm 1\right)^{2}$

Since the scaled normalization constants $\mathcal{M}_{1,2}$ should be positive we choose

$b_{2}=\left(\sqrt{b_{1}}-1\right)^{2}$

Thus we obtain 
$\mathcal{M}_{1}=\frac{1}{\sqrt{b_{2}}}=\frac{1}{\left|\sqrt{b_{1}}-1\right|}, \quad \quad \mathcal{M}_{2}=\frac{1}{\sqrt{b_{1}}}$

To conclude this section we note that in the case of $\mathcal{D} \neq 0$ we have two solutions for either $\mathcal{M}$, i.e.,

$\mathcal{M}_{1}^{(1,2)}=\frac{b_{2}-b_{1}+1 \pm \sqrt{\mathcal{D}}}{2 b_{2}}, \quad \quad \mathcal{M}_{2}^{(1,2)}=\frac{b_{1}-b_{2}+1 \pm \sqrt{\mathcal{D}}}{2 b_{1}}$.

Note also that the discriminant $\mathcal{D}$ is invariant (does not change) under permutation of $b_{1}$ and $b_{2}$. In other words, four different physically realizable situations are possible for a wide range of parameters

$0<b_{2}<1+b_{1}$

The inequality in Eq. (131) has been obtained under the condition that both solutions in Eq. (130) are positive. This case corresponds to the so-called "flip-flop" state ${ }^{17}$ of the two colliding beams, which is a bifurcated state better to be avoided.

\section{CONCLUSIONS}

We have developed a systematic canonical perturbation approach that removes rapidly oscillating terms in Hamiltonians of quite general form. The essential feature of this approach is the use of mixed canonical variables. For this purpose the Vlasov-Poisson equations are transformed to mixed canonical variables, and an appropriate perturbation scheme is chosen to obtain the equilibrium phase space density. It is worthwhile to note that the perturbation expansion outlined in the preceding section can be carried out to arbitrary order, although higher-order calculations become very tedious.

In conclusion, it is evident from the present analysis that the Hamiltonian averaging technique developed here represents a powerful formalism with applications ranging from beam propagation through a periodic focusing lattice (Sec. 3) to coherent beam-beam interaction (Secs. 4 and 5). For example, in the application to the coherent beam-beam interaction, the rapidly oscillating terms due to the periodic beam-beam kicks have been 
averaged away, leading to a new time-independent Hamiltonian (Sec. 4). Furthermore, the equilibrium distribution functions have been obtained as a general function of the new Hamiltonian, and coupled set of integral equations for the beam densities has been obtained (Sec. 5). An intriguing feature of the analysis in Sec. 5 is the derivation of a condition for

existence of the so-called "flip-flop" state ${ }^{17}$ of the two colliding beams, which is a bifurcated state better to be avoided in experimental applications.

We reiterate that the formalism developed here is strictly speaking non-canonical and in general does not provide complete elimination of fast oscillating terms in the transformed Vlasov equation in the mixed variables. Nevertheless, such an elimination can be performed in the new Hamiltonian in the mixed variables. Moreover, if the distribution function is assumed to be an arbitrary function of the new time-independent Hamiltonian, it is a stationary solution of the nonlinear Vlasov equation in the mixed variables.

Finally, we reiterate that the mixed variable Hamiltonian formalism developed in the present analysis can be used to derive amplitude equations, describing processes of formation of patterns and coherent structures in a number of plasma and beam systems in which collective processes are important.

\section{ACKNOWLEDGMENTS}

We are indebted to S.A. Heifets for many fruitful discussions concerning the subject of the present paper. It is a pleasure to thank H. Qin for illuminating discussions and comments. This research was supported by the U.S. Department of Energy. 


\section{REFERENCES}

1. R.C. Davidson and H. Qin, "Physics of Intense Charged Particle Beams in High Energy Accelerators", World Scientific, Singapore (2001), and references therein.

2. A.W. Chao, "Physics of Collective Beam Instabilities in High Energy Accelerators", Wiley, New York (1993).

3. S.I. Tzenov, "Collision Integrals and the Generalized Kinetic Equation for Charged Particle Beams", FERMILAB-Pub-98/287, Batavia (1998).

4. Y. Cai, A.W. Chao, S.I. Tzenov and T. Tajima, Physical Review Special Topics on Accelerators and Beams 4, 011001 (2001).

5. A.W. Chao, P. Bambade and W.T. Weng, in Proceedings of the US-CERN School on Particle Accelerators, Santa Margherita di Pula, Sardinia, 1985, edited by J.M. Jowett, M. Month and S. Turner, Lecture Notes in Physics, Vol. 247, Springer-Verlag, Berlin (1986), p. 77.

6. J.T. Seeman, in Proceedings of the US-CERN School on Particle Accelerators, Santa Margherita di Pula, Sardinia, 1985, edited by J.M. Jowett, M. Month and S. Turner, Lecture Notes in Physics, Vol. 247, Springer-Verlag, Berlin (1986), p. 121.

7. P.J. Channell, Physics of Plasmas 6, 982 (1999).

8. R.C. Davidson, H. Qin and P.J. Channell, Physical Review Special Topics on Accelerators and Beams 2, 074401 (1999); 3, 029901 (2000).

9. S.I. Tzenov and R.C. Davidson, "Hamiltonian Formalism for Solving the Vlasov-Poisson Equations and Its Application to the Coherent Beam-Beam Interaction", in Proceedings of the 2001 Particle Accelerator Conference, in press (2001).

10. S.I. Tzenov, "Contemporary Accelerator Physics", World Scientific, Singapore, in preparation (2001).

11. S.I. Tzenov and R.C. Davidson, "Macroscopic Fluid Approach to the Coherent BeamBeam Interaction", in Proceedings of the 2001 Particle Accelerator Conference, in press (2001). 
12. A.W. Chao and R.D. Ruth, Particle Accelerators 16, 201 (1985).

13. M. Abramowitz and I.A. Stegun, "Handbook of Mathematical Functions with Formulas, Graphs, and Mathematical Tables", Wiley, New York (1984).

14. J. Haissinski, Nuovo Cimento 18B, 72 (1973).

15. Yu.L. Klimontovich, "Statistical Physics", Harwood Academic Publishers, Chur (1986).

16. A.H. Nayfeh, "Introduction to Perturbation Techniques", Wiley, New York (1981).

17. A.V. Otboyev and E.A. Perevedentsev, Physical Review Special Topics on Accelerators and Beams 2, 104401 (1999). 